\title{
Shopping for Clothes: From Meeting Individual Needs to Socializing
}

\author{
Pratitee Sinha, Arwa Alsubhi, Saroj Dash, Lijie Guo, Bart P. Knijnenburg \\ Clemson University School of Computing \\ 215 McAdams Hall, Clemson SC 29634 \\ psinha@clemson.edu, aalsubh@clemson.edu, sdash@clemson.edu, lijieg@clemson.edu, bartk@clemson.edu
}

\begin{abstract}
In this paper, we analyze how shopping for clothes continues to be a social phenomenon, despite technological advances. Using a grounded theory approach, we coded 16 semi-structured interviews to develop process models of online and in-store shopping and to identify the constraints of each method. We then analyzed apparel shopping from the lens of Engeström's model of Activity Theory to compare online and in-store shopping at a conceptual level. We offer design recommendations for building systems and processes that bridge the gap between online and offline shopping.
\end{abstract}

Clothes, shopping, cognition, activity theory, Human-Computer Interaction.

\section{INTRODUCTION}

Clothes are an indispensable part of our daily lives. They have both a functional and a social role, representing one's identity, gender, culture, and status. This motivates our research into how people choose what to wear, the factors that influence their choices, and how and where they buy their clothes. Using a grounded theory approach, we interviewed people about apparel shopping, and developed process models of off- and online shopping, highlighting the constraints of each method. We identified a pervasive reliance on social support in both off- and online shopping, albeit in different forms.

We then generalized our understanding of shopping using Activity Theory, which takes socio-cultural context into consideration in describing the performance of tasks in collaboration with other people, tools, and the environment. Using this theory, we expose tensions in the socio-technical system that may underlie some of the typical dissatisfactions associated with online shopping. This allows us to formulate design recommendations that enable designers to build systems and processes that bridge the gap between online and offline shopping.

\section{RELATED WORK}

Although a growing number of people have migrated to online shopping for most of their needs, buying instore is still popular, (Kacen et al., 2013) especially for clothes. Why do people prefer traditional stores? Prior work has focused on the importance of communication. Guo et al. (2011) studied an instant messaging tool used by Chinese e-marketplace
Taobao, and showed that communication between buyers was a fundamental driver of purchasing activity. Similarly, Muta et al. (2014) found that systems facilitating collaborative shopping support information-sharing and discussion.

Monsuwé et al. (2004) described the need for interaction with a salesperson as a personality characteristic of a shopper. Shopping websites should provide such people with a means to interact with the store, so that they feel at ease placing orders. Studies on electronic word of mouth (eWOM), have focused on developing inference space models to present shoppers with relevant information from comments and reviews, which promotes interaction (Fujimoto, 2012). The current paper analyzes eWOM from the perspective of Activity theory, and compares its relevance with the offline counterpart.

\section{METHODS}

\subsection{Subjects}

16 participants (8 females) were recruited using snowball sampling. 3 were undergraduate students at Clemson University, 12 were graduate students, 1 non-student. The participants came from different cultures; 3 Arab, 7 Indian, 3 Chinese, and 3 American. Participants' ages ranged from 21 to 33 . 3 participants were married, 2 of them had children.

\subsection{Procedures and interview questions}

Participants answered open-ended questions, and were asked to show us how they shop online. They were asked to think aloud, to help us understand 
their actions. We joined one participant to shop at a store. Interviews were recorded, and we took extensive notes regarding participants' behaviors.

Participants were first asked about their preferred way to shop for clothes. Beyond that question, interviews were mostly driven by participants' responses. We also asked them to recall the procedures and decisions involved in specific recent instances of buying clothes online, how they felt when receiving package, and the further course of action if the item disappointed them.

We also asked participants about the kinds of clothes they bought, what factors influenced their decisions to adopt on- or offline shopping for each type of clothes, and the procedures they followed for buying each type of garment. Participants "steered" the interviews, but we sought to answer the following questions: What influences people to shop online versus in-store? Why do they rely on reviews and ratings in online shopping? What aspects of in-store shopping do they miss in online shopping? What causes dissatisfaction in online shopping?

\subsection{Analyzing the collected data}

Constructivist grounded theory guided our interviews and analyses (Charmaz, 2014): we iteratively coded 3-4 interviews, and then used gathered insights to guide further interviews. Coding started by assigning categories to the collected data. We then compared codes to find common or contrasting phenomena across participants, and further refined the codes. This methodology allowed us to develop theories iteratively and from the ground up.

We first developed process models of online and instore shopping, highlighting the constraints of each method. Next, we covered users' reliance on social support. Finally, we applied Activity Theory to our findings. In line with the grounded theory methodology, this higher-level theory was applied after the development of a grounded theory.

\section{OVERVIEW OF APPAREL SHOPPING}

\subsection{Online shopping as a side activity}

Our participants associated online shopping with a greater amount of comfort than shopping in stores, because it offers the ability to shop while doing other activities. For instance, one participant stated:

\footnotetext{
"I don't consider myself shopping, because I am doing other stuff when I am shopping online. Like at home I am baking something and also looking for stuff online, so my whole time isn't committed to shopping online."
}

This type of casual shopping is further facilitated by the proliferation of mobile applications of popular online shopping websites, which allow users to shop as a side activity anywhere and anytime. Shopping in stores, on the other hand, requires shoppers to set aside some time to go to the store. Once inside the store, they are totally involved in the process of shopping and cannot accommodate any other task.

In sum, in online shopping, time is shared: shoppers can do it while doing other activities. But in shopping in stores, time is dedicated: shoppers are fully involved with the process until it is completed.

\subsection{The Bittersweet Feeling of Time Delay}

Figures 1 and 2 illustrate the steps involved in the on- and offline shopping process, respectively. Online purchases take more time than in-store purchases, because there is an additional waiting period associated with shipping (WAITING TIME 1 in figure 1) which inadvertently makes online shopping slower than its in-store counterpart.

Asking our participants about this time delay, we were surprised to hear that they were not resentful about it. One participant described a mixed feeling of longing and suspense during the waiting time. This feeling was preceded by a phase of excitement when the order was placed, and was followed by another phase of excitement when the order was delivered. The participant explained:

\begin{abstract}
"There is a sort of two parts excitement to it. You are excited when you order it and then there is sort of suspense of waiting for it and then you get it and it's like Christmas and you are getting a good gift, a present that you bought for yourself."
\end{abstract}

We found that the primary reason for disappointment with online shopping was not the first but the second delay associated with returning the item (WAITING TIME 2 in figure 1). When shoppers are not satisfied, they suffer discontentment and regret as they have to return the item. While this happens for both online and in-store purchases, our participants claimed that of returning an online purchase takes longer and is more frustrating than returning an item to a store. Instore shoppers can return or replace an item quickly and easily, but this process is complicated and stressful for online purchases. The shopper must place a return request, print a return label. re-pack the clothes, and go to the post office or have the item picked up. Then, they must wait to get the refund or replacement. One of our participants said:

\footnotetext{
"I bought clothes for my kids, and when I got them it was too big for them. I did not return it because returning takes so long. [...] That is the reason I prefer shopping in malls. If you want to return something you have 30 days and I can drop it off any time."
}

We describe this as a "bittersweet feeling" because online shoppers feel extra happy when they receive the product, but also extra disappointed when the product does not fit and they have to return it. 


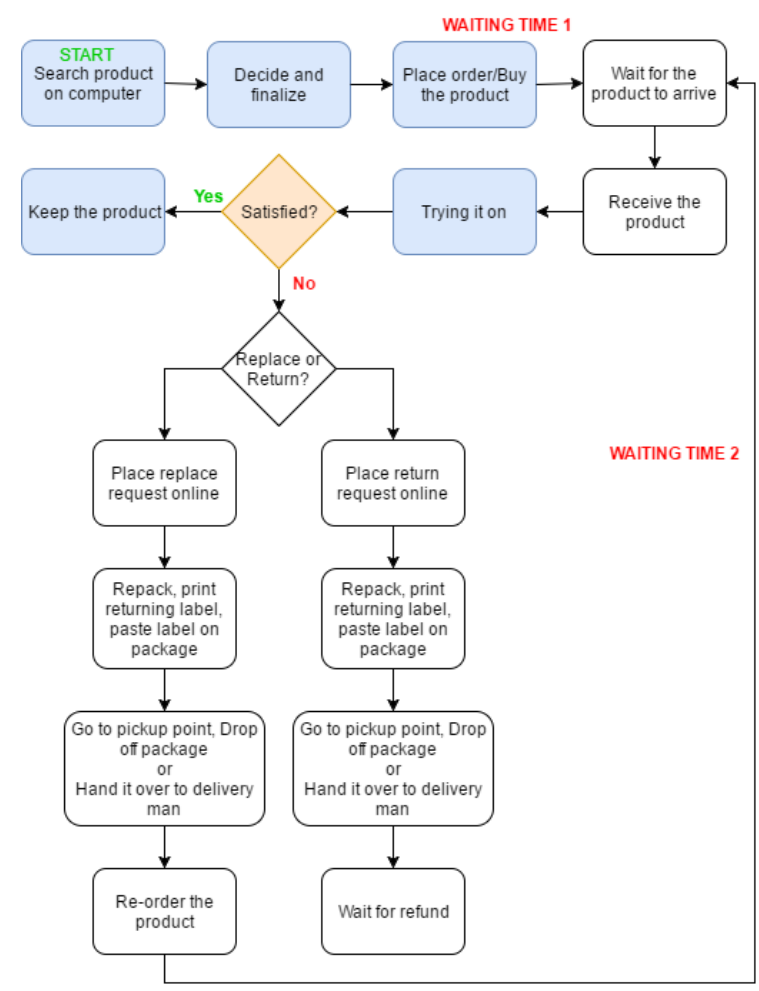

Figure 1: Steps taken purchasing clothes online.

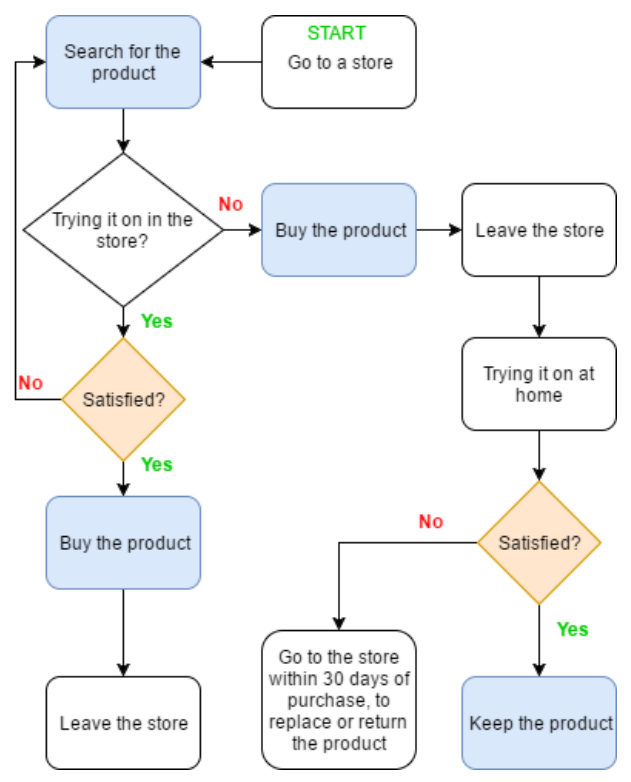

Figure 2: Steps taken purchasing clothes in-store.

\subsection{Experiencing Choice Overload}

While some participants mentioned that the wealth of options online caused contentment and delight, others argued it caused confusion, and dismissed online shopping as overwhelming. To combat this choice overload, shopping sites allow users narrow down their search. This creates satisfaction for some people, because it allows them to find items that align exactly with what they want. In fact, it makes finding an item with particular specifications online a lot more convenient than in-store. In one of the interviews, our participant explained:
"For my bridesmaid dress, my sister-in-law told us what type of dress we were looking for and so it was easier to choose the dress online than finding a store that had this specific style because that specific style was difficult to find in store."

At the same time, the plethora of choices invariably caused confusion and dissatisfaction with online shopping when participants had no specific requirements. One participant told us that she would add clothes to her online shopping cart, but not being able to choose among them, she would end up not buying any of them. This is a typical case of choice overload, which leads to disapproval of the system.

In sum, online shopping provides an abundance of options and filtering/sorting capabilities that are beneficial when shoppers have specific requirements, but overwhelming when they are simply browsing the collection.

\subsection{Shopping for casual and occasion wear}

We noticed that participants shop for two major categories of clothes: casual wear and occasion wear. Casual wear is clothes that people wear every day, like shirts, t-shirts, jeans, shorts, and dresses. Occasion wear is generally more expensive, and is bought for special events like formal meetings, parties, get-togethers, and weddings.

For special events, participants tended to pay a lot of attention to their attires. This urged them to try clothes on before buying them, which is why they would usually shop for occasion wear in physical stores, often accompanied by a person whose sense of style they trust. Participants avoided shopping online for occasion wear, because of the difficulty of visualizing the fit and style. Participants were also worried about buying an expensive item before getting to judge its quality. The hardship of returning an item if they are dissatisfied was a major deterrent in buying occasion wear online. One participant stated:
"For online, if it is very expensive, I have to spend time checking the return policy and if I buy something that doesn't fit me well and it is pretty expensive I have to again go through the hassle of returning the item".

Participants were worried about whether they would get the replacement in time before the event. Aside from the hassle of making the return, this would add additional difficulty of finding an alternate outfit.

For casual wear, some participants shopped in-store while others shopped online. Male participants preferred shopping online, because it involved less effort than to going to a mall. One participant said he would first see if he could get the item he was looking for online, but then check the shipping time and his need for the garment to decide whether he would venture to the store or place the order online. 


\subsection{Situational constraints}

We identified constraints that consistently served as deterrents for online or offline shopping (Table 1). Since most are situational rather than structural, it is unsurprising that none of our participants always shopped online or always in-store; the most convenient method depended on the situation.

Table 1: Online and Offline Shopping constraints.

\begin{tabular}{|l|l|}
\hline \multicolumn{1}{|c|}{ Online constraints } & \multicolumn{1}{c|}{ Offline constraints } \\
\hline Price, including shipping & Distance to the store \\
\hline Shipping time & Availability of transportation \\
\hline Website usability & Store hours \\
\hline Return policy & Traffic conditions \\
\hline & Availability of parking space \\
\hline & Schedule for the day \\
\hline
\end{tabular}

\section{SHOPPING - A SOCIAL PHENOMENON}

\subsection{Shopping to socialize}

Although shopping in stores requires planning and time commitment, our participants regarded it not just as a transactional process of buying things, but as a social activity, and they made an extra effort to set time aside for this engagement:

\begin{abstract}
"I usually go shopping with my best friend, so I spend a lot of time with her, it is kind of like hanging out together. That is another way of enjoying ourselves, we can have dinner or dessert together, and it is not just about shopping for clothes."
\end{abstract}

"I go to the mall to have some fun especially if I have friends or relatives who are coming from different towns or countries to visit me. I take them to the malls. We shop and eat. Also, our kids play together in the playground."

Participants valued the opinions of a close friend or family member, especially regarding occasion wear. These "co-shoppers" had a social influence, and were sometimes trusted for their fashion sense. Coshopping is best supported in-store, where the shopper tries the outfit and the co-shopper evaluates their appearance. When asked if she liked being accompanied while shopping, one participant responded:

\footnotetext{
"I would say yes, especially my mom because she loves fashion more than me. When I shop with her, she really gives good advice, because she knows me better than other people."
}

This process creates an environment of bonding that is difficult to replicate online. One participant mentioned that just walking around the shopping mall with her friends is a delightful experience, which contributes to her preference for shopping in-store.

Some participants partially facilitated the social aspects when shopping online by sending links to the co-shopper and receiving feedback via instant messaging or email. This process often results in a delay. If the co-shopper is at the same location, they can share the computer to give feedback. However, the sense of bonding is diminished here, and replaced by a task-oriented frame of mind.

We went shopping with one participant, which really helped us understand the role of the co-shopper. Constructivist grounded theory allows researchers to take an active role in the research setting, and the participant eagerly made use of this opportunity. Specifically, she took suggestions from us regarding the fit, size, and color of a jacket. She later explained that we really helped her buy the jacket. This shows that shoppers are happy to turn even our study into an opportunity to socialize and receive feedback while shopping.

\subsection{Gifting}

Exploring other social shopping experiences, we noticed that buying clothes as gifts is complicated due to the difficulty of estimating the right size. Gifting a gift card avoids this difficulty. One of our participants explained:

"I always prefer gifting something that is not related to size such as perfumes, scarfs... I gifted a gift card to my friend's child, because that's easier for me as I don't have to choose the size."

Another way to tailor gifts to the recipients' needs is the use of a gift registry. One participant explained:

\begin{abstract}
"My friend got married three weeks ago, she had a registry online for her wedding. [...] So I chose what I could afford from that list. [...] Then that item came out of the registry, and no one else bought the same thing for her. This is a good way to gift as you get what you want without receiving the same thing from many friends. It is also easy for us as friends when we are looking for something to gift someone."
\end{abstract}

This system facilitates a collaborative shopping activity across a group of people. It makes deciding what to buy more convenient, empowers the receiver of the gift control what she receives, and prevents duplicate gifts. Such a system could also facilitate the process of gifting clothes, where the recipient selects the correct items, colors and sizes.

\subsection{Trusting Reviews and Comments}

Reviews allowed participants to gauge quality and fit, and thereby helped to narrow down their choices. Reviews particularly acted as a deciding factor when the brand was not well known and the shopper had to decide whether to trust it. Reviews and ratings open a channel with feedback from other users who have bought the same product. As such, they mirror the social aspect of shopping with a co-shopper. We found that participants analyzed reviews carefully, and tried to understand the context in which the 
reviews had been written, especially in case of bad reviews. One interviewee mentioned:

\begin{abstract}
"if the comments are about the delivery not being good and I am not so worried about delivery time right now then I would still go ahead and buy that. But if the comments are about quality then I would steer clear of it."
\end{abstract}

Participants also spent time investigating the authenticity of the reviews. One said he would get suspicious about items with inconsistencies in ratings and reviews, while another expressed suspicion when he encountered many very good reviews, because it made him wonder whether the seller is writing these reviews themselves. Due to the diversity of our participants, we could deduce that this caution seemed to vary by culture. Participants from China and India were particularly wary about fake reviews, possibly because the occurrence of online fraud has risen in Asian countries (Ou, Sia and Banerjee, 2007). One Asian participant had developed a strategy purchasing from the Indian e-commerce giant Flipkart: He only relied on the ratings and reviews of "certified buyers" who have actually purchased the product. Another participant mentioned they trusted the reviews on very popular websites such as Amazon.

Thus, users extensively use reviews and ratings in their purchasing decisions. To make sure that they are not unduly influenced, they filter them based on apparent legitimacy and how relevant they are.

\section{4. (Not) Trusting Salespersons}

Caution regarding online reviews seemed to spill over to in-store advice. Two participants from India stated that they prefer online shopping without getting influenced. They mentioned how salespersons used marketing strategies and tried to convince them to buy their products, which caused confusion, and disoriented them from their target.

This interesting observation shows that not all participants appreciate just any kind of social interaction while shopping. While shoppers appreciate the advice of a co-shopper, they are annoyed when they feel pressured by a salesperson.

\subsection{Being influenced by family advice}

We also came across a strong influence of family members in certain cultures. We interviewed two Middle Eastern women, both of whom were always influenced by either a spouse or parent to choose modest clothes. In addition, an Indian participant mentioned that shopping for wedding clothing is an elaborate process, which involves not only buying different outfits for each day for the bride and groom, but also buying clothes as gifts for both families. Thus, a group of family members usually venture out for a shopping process that can span several days.

\section{SHOPPING THROUGH THE LENS OF ACTIVITY THEORY}

We established that shopping for clothes is strongly influenced by cultural and societal forces. We therefore decided to interpret our findings through the lens of Activity Theory. In this context shopping for clothes is a recurring activity, and shoppers' plans are anticipatory reflections of this activity (Bardram, 1997). One of our participants-an avid online shopper-talked about her plans:

"First I search the brand name, and then I see the rating, like say four or five stars, and then I see how many people have purchased this product. And the last thing I may want to look through is the reviews."

We subsequently analyzed the online and in-store shopping process with the help of Engeström's Activity System Model (2000). Figure 3 shows the model. The shopper, who is the subject in this Activity System, aims to select an outfit (object or goal), leading to the outcome of buying clothes. For in-store shopping, this process is assisted by artifacts such as transportation to the store (which may not be available), the shopper's time schedule (which may prevent them from doing an elaborate search) and her prior knowledge (e.g., she may have experience in feeling fabric to judge its quality, or she may know the layout of the store). Co-shoppers are the community assisting her in selecting the right outfit. Thus, the shopper and the co-shoppers divide the labor of the decision-making process in this model. The entire activity is governed by the decorum of shopping, which varies from one culture to another, and this serves as the rules in this model.

Switching to online shopping, the computer acts as an important mediating artifact, which gives online shopping an advantage in terms of availability (both in terms of physical presence, and its ability to efficiently filter items), but the rules are additionally governed by the shipping and return policies of the website. The time delay caused by these rules leads to a tension in this model, as it sometimes hinders the shopper from achieving her goals (i.e. getting the item delivered in time for an event). The computer also creates a barrier to co-shopping, and users replace this community by reviews and ratings. In terms of division of labor, as users increasingly shop online, they tend to increasingly rely on reviews and ratings in their decision-making process. This dependency on online reviews creates an apparent tension within the model. As discussed above, some participants questioned the legitimacy of the reviews, which led them to spend more time contemplating whether to trust them or not. Moreover, the community of online reviewers may not always reflect the values of the (arguably) closer community of co-shoppers, which leads shoppers to carefully analyze the context of each review. 


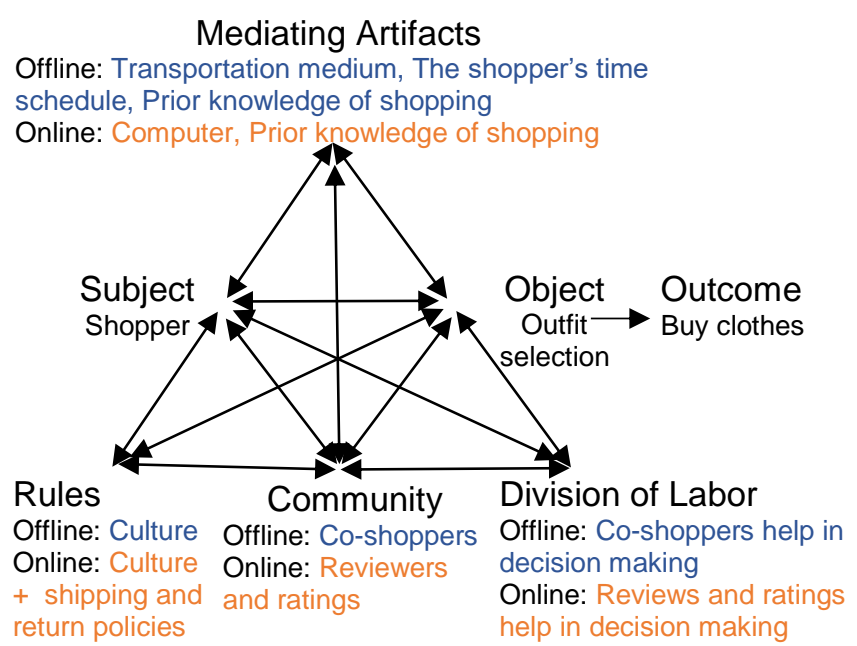

Figure 3: Activity model of buying clothes online and in stores.

\section{DESIGN RECOMMENDATIONS}

We have several recommendations to address the tensions in the model described above. One suggestion is a body calibration recommendation system that leverages reviews of people who suggest picking a larger or smaller size to calculate the best size. An advanced version could even develop a model of users' body dimensions based on their past orders and returns, and then recommend the size most ordered by other users with similar body dimensions.

Another idea resolves the tension of distrust in reviews. Existing research demonstrates the ability to identify legitimate reviews (Banerjee et al., 2015), and some e-commerce giants help users by highlighting the reviews of actual buyers. However, more work needs to be done to help users to quickly identify legitimate and relevant reviews. One solution is to reintroduce co-shoppers into the division of labor. This feature could allow users to post options to their social network, and ask friends and family to post feedback. While most online retailers already provide functionality to post products to social media, it is usually not marketed as a tool for socially supported decision-making.

Finally, shoppers resolve the tension of time availability by switching between on- and offline shopping, and this switch can be made more fluent. For example, a store could allow users to filter their online catalogue to identify a set of eligible items, and then allow them to "reserve" these items for an in-store fitting. This combines the efficiency of online filtering with the assurance of trying the clothes in-store.

\section{CONCLUSION}

In this paper, we investigated how people shop for clothes, and framed it through the lens of Activity Theory. Participants valued online shopping for its efficiency, and in-store shopping for the opportunity to socialize and get feedback from co-shoppers. As co-shopping is difficult online, it is replaced by inspecting reviews and comments. Shopping sites have been trying to replicate the offline process by incorporating interaction with others during the shopping process, and we have identified future directions to further facilitate such interaction. For example, we believe that social networking can bring some socializing activity to online shopping as well.

Conversely, users can shop online as a side activity, and the availability of filtering functionality allows them to more efficiently find items that fit certain requirements. We believe that a hybrid functionality may allow users to attain the best of both worlds.

\section{REFERENCES}

Banerjee, S., Chua, A. Y. K. and Kim, J.-J. (2015) 'Using Supervised Learning to Classify Authentic and Fake Online Reviews', IMCOM '15.

Bardram, J. E. (1997) 'Plans as Situated Action: An Activity Theory Approach to Workflow Systems', ECSCW'97, pp. 17-32.

Charmaz, K. (2014) Constructing Grounded Theory. SAGE.

Engestrom, Y. (2000) 'Activity theory as a framework for analyzing and redesigning work', Ergonomics, 43(7), pp. 960-974.

Fujimoto, K. (2012) 'Investigation of Potencymagnitude Relations of eWOM Messages with a Focus on the Distinction between Attitude Direction and Strength', IAT'12, pp. 69-73.

Guo, S., Wang, M. and Leskovec, J. (2011) 'The Role of Social Networks in Online Shopping: Information Passing, Price of Trust, and Consumer Choice', EC'11, pp. 157-166.

Kacen, J. J., Hess, J. D. and Chiang, W.-Y. K. (2013) 'Bricks or Clicks? Consumer Attitudes toward Traditional Stores and Online Stores', Global Economics and Management Review, 18(1), pp. 12-21.

Monsuwé, T. P. y, Dellaert, B. G. C. and Ruyter, K. de (2004) 'What drives consumers to shop online? A literature review', Intl. Journal of Service Industry Management, 15(1), pp. 102-121.

Muta, M., Mukai, K., Toumoto, R., Okuzono, M., Hoshino, J., Hirano, H. and Masuko, S. (2014) 'Cyber Chamber: Multi-user Collaborative Assistance System for Online Shopping', ITS'14, pp. 289-294.

Ou, C. X., Sia, C. L. and Banerjee, P. (2007) 'What is hampering online shopping in China', J. of Information Technology Management, 18(1), pp. 16-32. 
Shopping for Clothes: From Meeting Individual Needs to Socializing

Sinha • Alsubhi • Dash • Guo • Knijinenburg 